Article

\title{
Molecular Analysis of Canine Filaria and Its Wolbachia Endosymbionts in Domestic Dogs Collected from Two Animal University Hospitals in Bangkok Metropolitan Region, Thailand
}

\author{
Hathaithip Satjawongvanit ${ }^{1}$, Atchara Phumee 2,3(0, Sonthaya Tiawsirisup ${ }^{4}$, \\ Sivapong Sungpradit ${ }^{5}\left(\mathbb{D}\right.$, Narisa Brownell ${ }^{2}$, Padet Siriyasatien ${ }^{2}\left(\mathbb{D}\right.$ and Kanok Preativatanyou ${ }^{2, *}$ \\ 1 Medical Science Program, Faculty of Medicine, Chulalongkorn University, Bangkok 10330, Thailand \\ 2 Vector Biology and Vector Borne Disease Research Unit, Department of Parasitology, Faculty of Medicine, \\ Chulalongkorn University, Bangkok 10330, Thailand \\ 3 Thai Red Cross Emerging Infectious Disease-Health Science Centre, World Health Organization \\ Collaborating Centre for Research and Training on Viral Zoonoses, Chulalongkorn Hospital, \\ Bangkok 10330, Thailand \\ 4 Veterinary Parasitology Unit, Department of Veterinary Pathology, Faculty of Veterinary Science, \\ Chulalongkorn University, Bangkok 10330, Thailand \\ 5 Department of Pre-clinic and Applied Animal Science, Faculty of Veterinary Science, Mahidol University, \\ Salaya, Nakhon Pathom 73170, Thailand \\ * Correspondence: Kanok.Pr@chula.ac.th; Tel.: +66-2256-4387
}

Received: 4 June 2019; Accepted: 26 July 2019; Published: 29 July 2019

\begin{abstract}
Canine filariasis is caused by several nematode species, such as Dirofilaria immitis, Dirofilaria repens, Brugia pahangi, Brugia malayi, and Acanthocheilonema reconditum. Zoonotic filariasis is one of the world's neglected tropical diseases. Since 2000, the World Health Organization (WHO) has promoted a global filarial eradication program to eliminate filariasis by 2020. Apart from vector control strategies, the infection control of reservoir hosts is necessary for more effective filariasis control. In addition, many studies have reported that Wolbachia is necessary for the development, reproduction, and survival of the filarial nematode. Consequently, the use of antibiotics to kill Wolbachia in nematodes has now become an alternative strategy to control filariasis. Previously, a case of subconjunctival dirofilariasis caused by Dirofilaria spp. has been reported in a woman who resides in the center of Bangkok, Thailand. Therefore, our study aimed to principally demonstrate the presence of filarial nematodes and Wolbachia bacteria in blood collected from domestic dogs from the Bangkok Metropolitan Region, Thailand. A total of 57 blood samples from dogs with suspected dirofilariasis who had visited veterinary clinics in Bangkok were collected. The investigations for the presence of microfilaria were carried out by using both microscopic and molecular examinations. PCR was used as the molecular detection method for the filarial nematodes based on the COI and ITS1 regions. The demonstration of Wolbachia was performed using PCR to amplify the FtsZ gene. All positive samples by PCR were then cloned and sequenced. The results showed that the filarial nematodes were detected in 16 samples $(28.07 \%)$ using microscopic examinations. The molecular detection of filarial species using COI-PCR revealed that 50 samples $(87.72 \%)$ were positive; these consisted of $33(57.89 \%), 13(22.81 \%)$, and $4(7.02 \%)$ samples for D. immitis, B. pahangi, and B. malayi, respectively. While the ITS1-PCR showed that 41 samples $(71.93 \%)$ were positive-30 samples $(52.63 \%)$ were identified as containing D. immitis and 11 samples $(19.30 \%)$ were identified to have B. pahangi, whereas B. malayi was not detected. Forty-seven samples $(82.45 \%)$ were positive for Wolbachia DNA and the phylogenetic tree of all positive Wolbachia was classified into the supergroup $\mathrm{C}$ clade. This study has established fundamental data on filariasis associated with Wolbachia infection in domestic dogs in the Bangkok Metropolitan Region. An extensive survey of dog blood samples would provide valuable epidemiologic data on potential zoonotic filariasis in Thailand. In addition,
\end{abstract}


this information could be used for the future development of more effective prevention and control strategies for canine filariasis in Thailand.

Keywords: dog; filariasis; D. immitis; B. pahangi; B. malayi; zoonosis; Thailand

\section{Introduction}

Filariasis is a mosquito-borne parasitic disease, which is an important public health concern in tropical and subtropical areas. It affects approximately 886 million people in 52 countries worldwide [1]. The disease is commonly found in Southeast Asian countries [2]. Filariasis has been reported as an important zoonosis from dogs and cats, both of which are well recognized as reservoirs for filariasis [3]. Several major canine filarial species have been documented in several localities around the world, including Dirofilaria immitis, Dirofilaria repens, Brugia malayi, Brugia pahangi, Brugia ceylonensis, Brugia patei, Cercopithifilaria grassii, Acanthocheilonema reconditum, and Acanthocheilonema dracunculoides [4-6]. In Thailand, the common filarial worms in dogs are D. immitis, B. malayi, B. pahangi, and A. reconditum [7-9]. D. immitis is the most common species; it causes canine heartworm disease or canine dirofilariasis and is widespread. The disease is endemic in tropical and subtropical regions throughout the world [10]. Among the neglected tropical diseases, filariasis was selected as a particular target for achieving elimination by 2020, and this has been published in the World Health Organization (WHO) roadmap [11,12]. Previously, the elimination and control strategy of filariasis was based on the control of the mosquito vectors, such as those in the Mansonia, Anopheles, Aedes, Culex, and Ochlerotatus genuses $[13,14]$. However, animals, especially dogs, can also serve as the reservoirs of filarial nematodes. Therefore, the control of infection in all potential reservoirs is also essential. Moreover, a study in dog reservoirs might provide a more in-depth understanding of the patterns and epidemiologic data of filariasis, and it could also contribute to a decrease in the number of human cases. Zoonotic filariasis has been reported globally. In Thailand, several human cases of zoonotic filariasis have been documented. Some examples include the ocular Dirofilaria infections caused by Dirofilaria spp. [15] and D. repens [16] in the patients from Phangnga and Nakhon Si Thammarat provinces, respectively. The most recently reported case was in 2018. A 67-year-old woman residing in Bangkok presented with subconjunctival dirofilariasis. Molecular analysis of the sample obtained from the eye of the patient showed that it was caused by an unknown Dirofilaria spp. closely related to Dirofilaria hongkongensis [17].

Several reports suggest that filarial nematodes serve as hosts for an obligate bacterial endosymbiont, Wolbachia [18-20]. Wolbachia, a gram-negative intracellular bacterium, plays an important role in nematode biological development, reproduction, and survival. It also provides critical metabolites to the filarial nematode $[11,21,22]$. In addition, the correlation between Wolbachia-nematode symbiosis has provided treatment strategies for the control and eradication of the filarial infection in the host using Wolbachia as a target of antibiotic treatment [23,24]. Many studies show that filarial nematode in dogs can be diagnosed through the examination of circulating microfilariae [25-27]. However, when the Giemsa stain is used for characterization, it is difficult to differentiate and identify definite species among the closely related microfilaria species because of the similarities in their morphology. As previously mentioned, there is a report on the zoonotic case in Bangkok caused by an unknown Dirofilaria species, and the morphology identification problem of the blood stage nematode and the relationship between nematodes and Wolbachia remain unsolved. Therefore, the aims of our study are primarily to determine the presence of filaria nematodes in domestic dog blood samples from the Bangkok Metropolitan Region, Thailand using molecular techniques to precisely identify the species of detected filarial nematodes. Polymerase chain reaction (PCR) was used as a molecular technique to amplify the cytochrome c oxidase subunit I (COI) gene and internal transcribed spacer 1 (ITS1) region, which are both suitable molecular markers given the high degree of genetic variation in filarial nematodes. The study was also used to investigate the status of Wolbachia symbionts in filarial 
nematodes in Thailand. The presence of Wolbachia bacteria was demonstrated by PCR to amplify the filamenting temperature-sensitive protein mutant $Z$ (FtsZ) gene, which plays a role in the cell division of Wolbachia and can also be found in high copy numbers. Sequences obtained from the study were used for phylogenetic tree construction. Information obtained from the study provides preliminary data on filariasis associated with Wolbachia infection in domestic dogs in Bangkok, Thailand. These data will be useful in the future for reservoir- and Wolbachia-based programs for filariasis control.

\section{Results}

\subsection{Morphological Characteristics of Filarial Worm}

Microfilariae were detected in 16 of 57 (28.07\%) dog blood samples collected in the Bangkok Metropolitan Region using the Giemsa staining technique. The morphological identification for microfilariae species found that 11 samples (19.30\%) were Dirofilaria spp. and 5 samples $(8.77 \%)$ were Brugia spp. (Table 1). For the Dirofilaria spp., Giemsa staining showed unsheathed microfilaria and the relative positions of the nerve ring (NR), excretory pore (Ex.P), excretory cell (Ex.C), first genital cell (G1), anal pore (AP), and the terminal nucleus (TN) at the tail. (Figure 1). For the Brugia spp., Giemsa staining showed clear sheathed microfilaria and one nucleus in the elongated cephalic space. The position of the nerve ring (NR), excretory pore (Ex.P), anal pore (AP), and two discrete overlapping terminal nuclei at the tail end are shown in Figure 1.

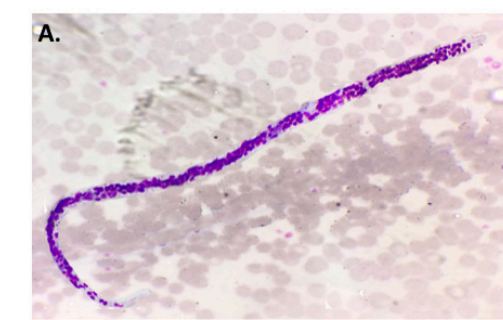

C.

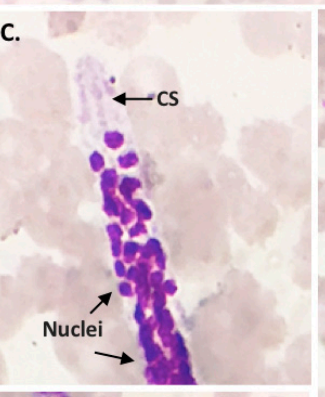

F.
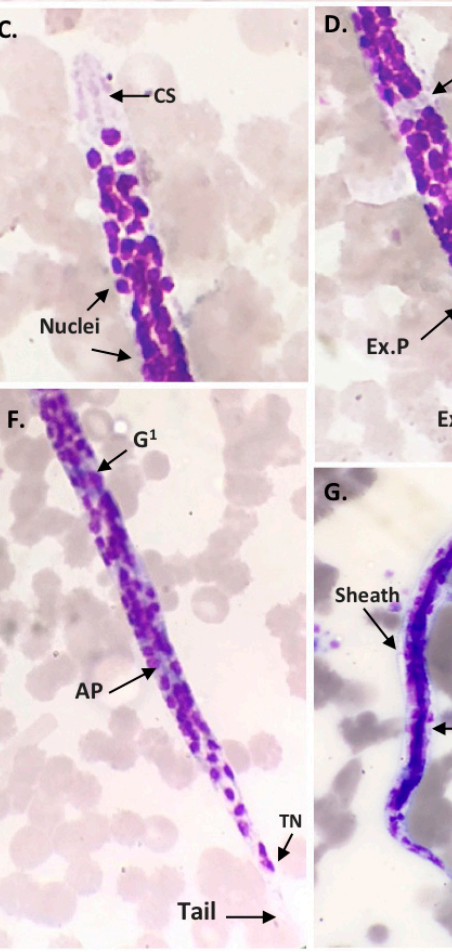

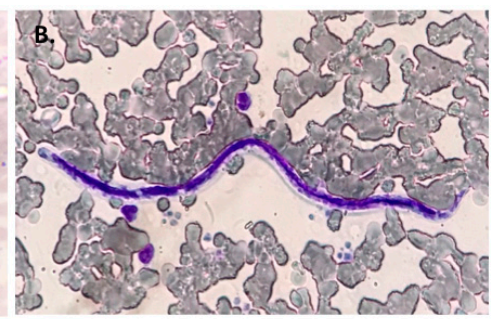

E.

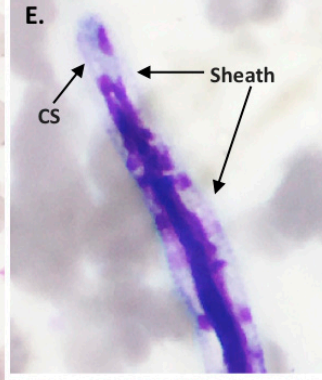

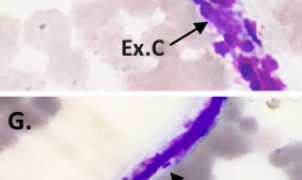

H. $\cos$.
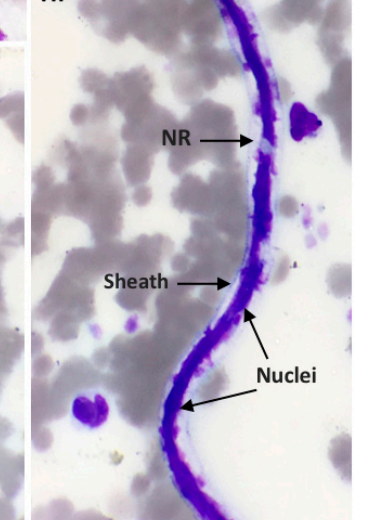

Figure 1. Microfilaria of Dirofilaria spp. (A,C,D,F) and Brugia spp. (B,E,G,H), Giemsa stain, 100×. The morphological marks show the position of several structures: The cephalic space (CS), nerve ring (NR), excretory pore (Ex.P), excretory cell (Ex.C), genital cell (G1), anal pore (AP), and terminal nucleus (TN). 


\subsection{Molecular Detection of Filarial Nematode}

Both COI-PCR and ITS1-PCR were able to detect filarial nematode DNA in the blood samples. Of the 57 blood samples collected, $50(87.72 \%)$ samples were amplified. The nucleotide sequences of the partial COI gene contained approximately $690 \mathrm{bp}$ (Figure S1) and were able to classify filarial nematodes into three species: D. immitis, B. pahangi, and B. malayi, accounting for $33(57.89 \%), 13(22.81 \%)$, and $4(7.02 \%)$ of the samples, respectively. Meanwhile, 41 samples $(71.93 \%)$ were amplified for filarial nematode DNA using PCR annealed specifically to the ITS1 region, which has approximately $600 \mathrm{bp}$ for D. immitis (52.63\%) and $550 \mathrm{bp}$ for B. pahangi (19.30\%) (Table 1, Figure S2). According to the sequence analysis of all sequences included in this study (Figures S3 and S4), the results of partial COI sequence comparisons between the species showed a sequence similarity percentage of $98.9 \%-100 \%$ (mean of $99.72 \%$ ), $98.2 \%-100 \%$ (mean of $99.05 \%$ ), and $98.6 \%-100 \%$ (mean of $99.22 \%$ ) for D. immitis, B. pahangi, and B. malayi respectively, whereas the sequence similarity of ITS1 was $83.3 \%-100 \%$ (mean of $92.2 \%$ ) and $96.3 \%-100 \%$ (mean of $98.3 \%$ ) for D. immitis and B. pahangi, respectively. The phylogenetic tree based on the partial COI and ITS1 sequence showed that all isolated samples from D. immitis clustered together in one group. Two isolated from B. pahangi and B. malayi were clustered together, and they were clearly separated branches despite being placed in the same genus (Figures 2 and 3). The partial nucleotide sequences of the COI and ITS1 obtained in this study were deposited in the GenBank under accession numbers MK250707-MK250757 and MK250758-MK250799, respectively. Based on the results, COI-PCR could be better suited for filarial species identification than ITS1-PCR. Additionally, present studies showed that COI evolves much more quickly than ITS1, revealing low intraspecific and interspecific molecular variability for filarial worms.

Table 1. The results of microscopic examinations and PCR for the detection of filarial worm DNA from blood dog specimens $(n=57)$. Positive controls used the DNA of Dirofilaria immitis, Brugia pahangi, and Brugia malayi.

\begin{tabular}{cccc}
\hline \multirow{2}{*}{ Species } & $\begin{array}{c}\text { Microscopic } \\
\text { Examination (\%) }\end{array}$ & \multicolumn{2}{c}{ Molecular Detection (\%) } \\
\cline { 3 - 4 } & & COI & ITS1 \\
\hline Dirofilaria spp. & $11(19.30 \%)$ & & \\
Brugia spp. & $5(8.77 \%)$ & & \\
D. immitis & & $33(57.89 \%)$ & $30(52.63 \%)$ \\
B. pahangi & & $13(22.81 \%)$ & $11(19.30 \%)$ \\
B. malayi & & $4(7.02 \%)$ & 0 \\
\hline Total & $16(28.07 \%)$ & $50(87.72 \%)$ & $41(71.93 \%)$ \\
\hline
\end{tabular}

\subsection{Wolbachia Detection Using Nested PCR}

Wolbachia DNA was detected based on the Wolbachia protein-coding housekeeping FtsZ gene (147 bp) (Figure S5). The result showed that 47 of 57 (82.45\%) blood dog samples were positive for Wolbachia DNA. The species detected consisted of D. immitis (57.89\%), B. pahangi $(19.30 \%)$, and B. malayi $(5.26 \%)$ (Table 2). The nucleotide sequence of FtsZ of the Wolbachia endosymbiont (Figure S6) had a similarity of $94.5 \%-100 \%$ (mean of $99.64 \%$ ). The phylogenetic tree based on the FtsZ gene revealed that 47 of the Wolbachia DNA samples in this study could be clearly classified into supergroup C (Figure 4). 
Table 2. Filarial worm tested for infection with Wolbachia using nested PCR of the FtsZ gene ( $\mathrm{n}=57$ ). Positive controls used the DNA of Wolbachia supergroup B in Aedes albopictus mosquitoes.

\begin{tabular}{cc}
\hline Species & Positive for Wolbachia (\%) \\
\hline D. immitis & $33(57.89 \%)$ \\
B. pahangi & $11(19.30 \%)$ \\
B. malayi & $3(5.26 \%)$ \\
\hline Total & $47(82.45 \%)$ \\
\hline
\end{tabular}

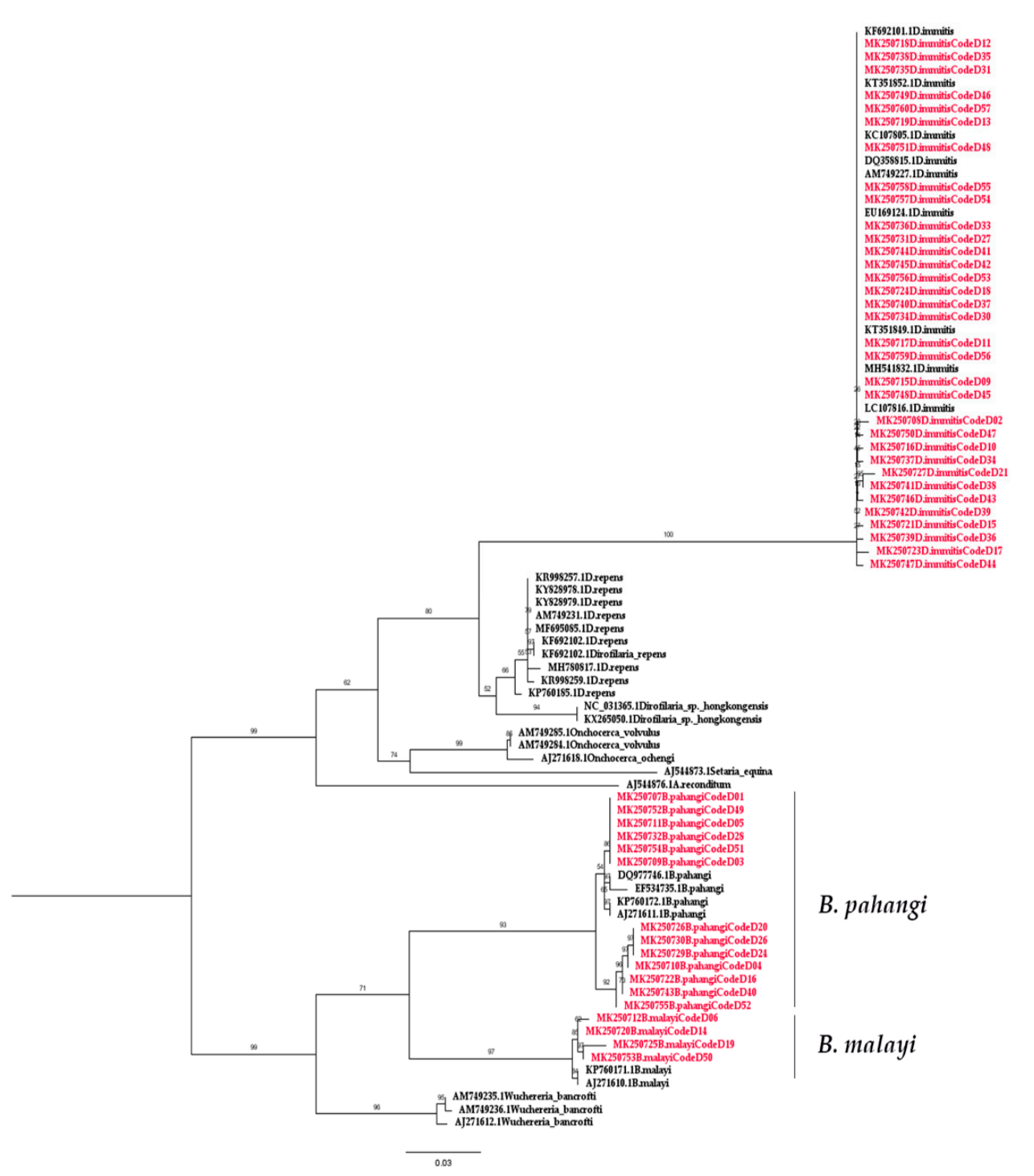

D. immitis

Figure 2. Phylogenetic tree of filarial worms constructed from the partial COI sequences. The red color represents the individual and combination identification sequences compared with reference isolates obtained from GenBank. Branch support was estimated based on 1000 bootstrap replicates. 


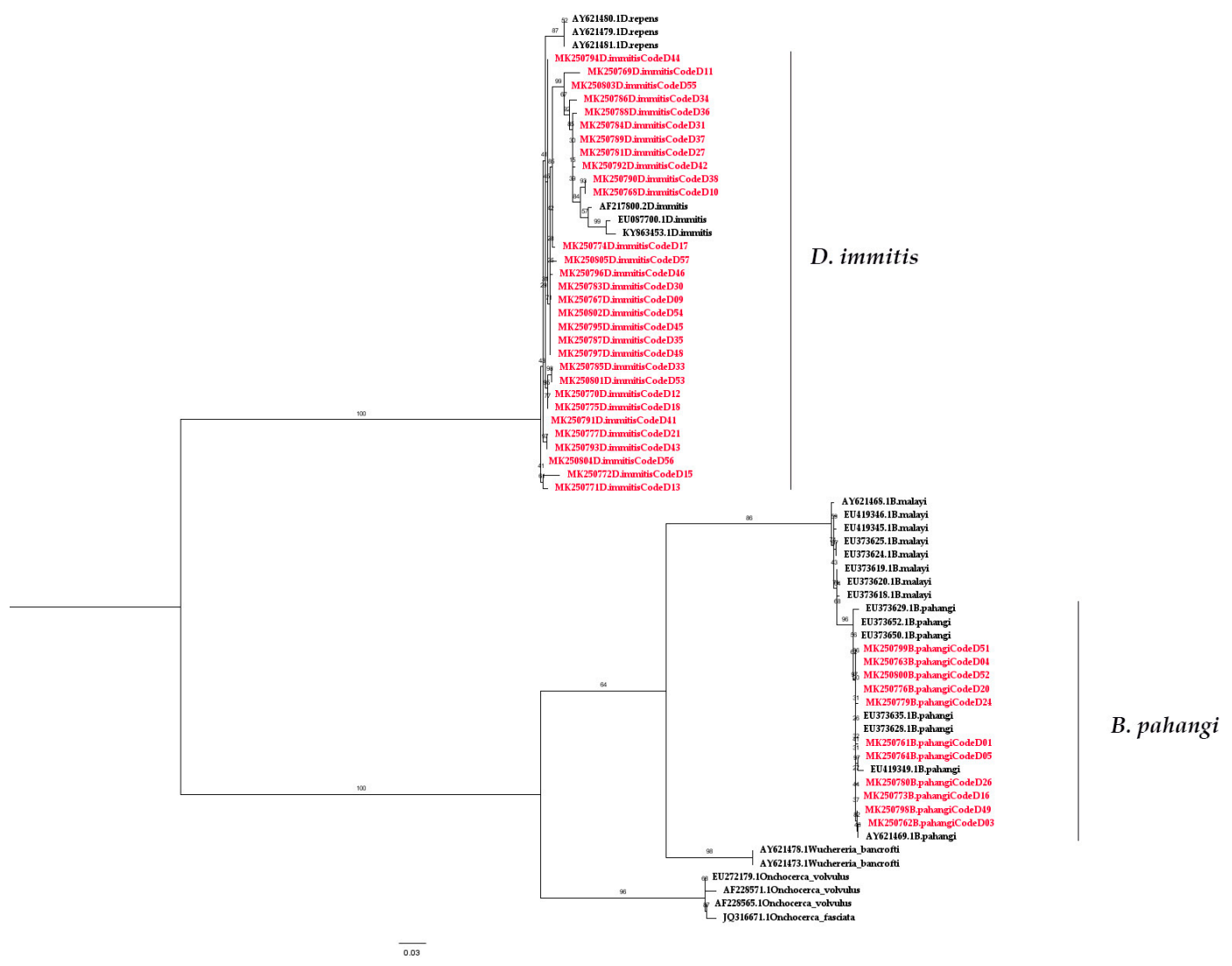

Figure 3. Phylogenetic tree of filarial worms constructed from ITS1 sequences. The red color represents the individual and combination identification sequences compared with reference isolates obtained from GenBank. Branch support was estimated based on 1000 bootstrap replicates.

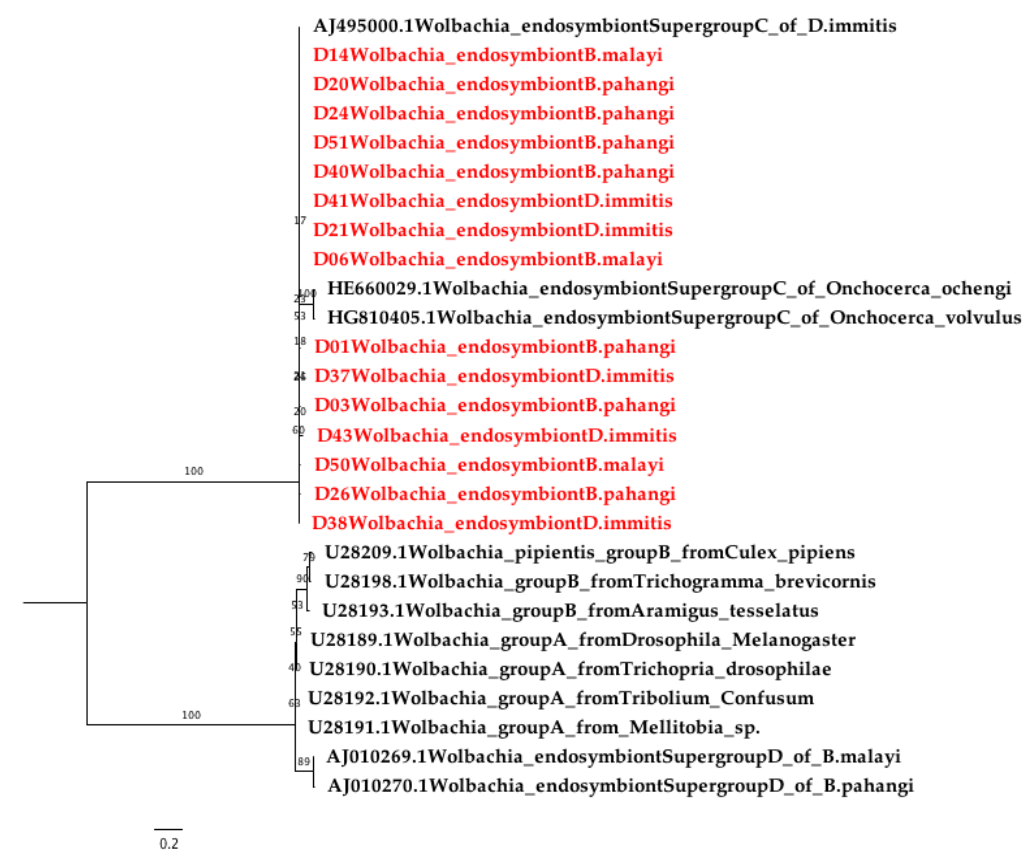

Supergroup C

Supergroup B

Supergroup A

Supergroup D

Figure 4. Phylogenetic tree of Wolbachia from filarial worms constructed from the partial FtsZ sequences. The red color represents the individual and combination identification sequences compared with reference isolates obtained from GenBank. Branch support was estimated based on 1000 bootstrap replicates. 


\section{Discussion}

Canine filarioids are important nematodes that are transmitted to vertebrate hosts through arthropods and mosquitoes. Nematodes can cause severe disease in dogs and, potentially, transmit the disease to humans [28]. Control of canine filariasis through vector control and the early treatment of infected reservoir hosts is essential. Therefore, a highly specific and sensitive technique for the diagnosis of canine filarioids from an early stage of infection is necessary. Although the gold standard method for the diagnosis of microfilariae in a blood smear is microscopic examination on glass slides stained with Giemsa or hematoxylin and eosin [29,30], this technique requires considerable expertise, has low sensitivity, and cannot clearly discriminate among closely related species of filarial nematodes, such as D. immitis, D. repens, and D. reconditum or B. malayi and B. pahangi [31]. Serologic and molecular techniques with high sensitivity and specificity for filariasis diagnosis have been developed as additional methods for the detection of microfilariae [6,32-34]. In this study, microscopic examination gives a low positive result for microfilariae $(28.07 \%)$ compared to PCR. We investigated conventional PCR for both canine filarial and Wolbachia bacterial infections in dog blood samples. PCR could be used to increase the detection rate of microfilaria DNA and also to differentiate filarial parasites among closely related species. This study also suggests that $C O I$ sequences can act as useful genetic markers in distinguishing between D. immitis, B. pahangi, and B. malayi; however, ITS1 can detect only D. immitis and B. pahangi. Thus, the COI-PCR method is a more suitable method for the PCR-based detection of filarial nematode infections in dog blood specimens than ITS1-PCR. Therefore, we recommend using the COI gene for filarial detection because it is commonly used in the study of filarial DNA detection and has shown a large number of homolog sequences available in GenBank for the comparison and evaluation of results. Unfortunately, the limitation of this study was that the number of dog blood samples was quite low in number. To increase the accuracy of these assays, a larger sample size might be needed.

There are several reports on the prevalence of major D. immitis infection in dogs from various countries, including China [35], Korea [36], Iran [37], Portugal [38], Hungary [39], and Thailand [7-9,40]. In this current study, we also found that $D$. immitis is the most frequent species found in dog blood samples using both COI-PCR $(57.89 \%)$ and ITS1-PCR (52.63\%). This is followed by B. pahangi, detected in $22.81 \%$ of samples using COI-PCR and $19.30 \%$ of samples using ITS1-PCR. In contrast, B. malayi can be detected in only $7.02 \%$ of samples using COI-PCR. According to previous studies, both $B$. malayi and B. pahangi infections in dogs [41-43] and cats $[7,9,44]$ have been found in different regions of southern Thailand, such as the Narathiwat and Satun provinces [7-9]. However, the information on filariasis in dogs caused by B. malayi and B. pahangi in terms of their relationship with their hosts, geographic distribution, and association with human or veterinary diseases is quite limited. Our study showed that the blood samples from dogs in the Bangkok Metropolitan Region were infected with both B. malayi and B. pahangi. Therefore, we suspect that domestic dogs may be a potential reservoir for B. malayi and B. pahangi in Bangkok. Further surveys throughout the country to investigate the prevalence of $B$. malayi and B. pahangi infection in a greater number of dog samples are needed in order to investigate the exact role of dogs as the natural reservoir hosts of $B$. malayi and B. pahangi. Further investigations on the mosquito vectors are also needed for the development of control protocols in the future.

The preliminary study of the phylogenetic tree based on the partial COI sequences of B. malayi-positive samples showed they were clustered together with B. malayi from France (accession no. KP760171.1) and Italy (accession no. AJ271610.1) with short branch lengths. The B. pahangi sequences of $C O I$ were also clustered together with B. pahangi from Malaysia (accession no. DQ977746.1 and EF534735.1), France (accession no. KP760172.1), and Italy (accession no. AJ271611.1) with small branch lengths. This is also true for B. pahangi sequences based on the ITS1 region. According to the result of the phylogenetic tree of filarial worms constructed from partial COI sequences, two isolated samples from B. malayi and B. pahangi were in the same cluster and could be clearly separated. For this reason, the COI sequence was useful for identifying these congeneric species, and COI-PCR also 
showed better amplification for multiple species than ITS1-PCR in the present study. Oh et al. (2017) suggested that COI is the so-called "barcode" for the identification of filarial species diversity [45]. The strength of this study is that this method can be used as a screening and survey tool to study the molecular epidemiology of filarial infection among the high-risk population. In this study, we did not find $D$. hongkongensis as we expected. This might be due to the small number of samples, and other vertebrates may be the definitive host for $D$. hongkongensis. Interestingly, many filarial nematodes contain Wolbachia, an obligate bacterial endosymbiont, which plays an important role in the biological development of filarial nematodes and is involved in targeted therapy against them, leading to the loss of worm fertility and viability upon antibiotic treatment $[23,24]$. Wolbachia can be classified into at least seven supergroups based on the FtsZ and Wolbachia surface protein (wsp) sequences [46-49]. Wolbachia supergroups $C$ and $D$ have been detected in nematodes, whereas four supergroups (i.e., A, B, E, and H) have only been found in arthropods [50]. Furthermore, supergroup F has been found in both arthropod and nematode species [47]. Our results showed the preliminary study of the phylogenetic tree of all positive Wolbachia from $33(57.89 \%), 11(19.30 \%)$, and $3(5.26 \%)$ samples of D. immitis, B. pahangi, and B. malayi, respectively. These were classified into a clade of supergroup C, which is closely related to the Wolbachia endosymbiont of D. immitis from Italy (accession no. AJ495000) and the Wolbachia endosymbiont of Onchocerca ochengi (accession no. HE660029) and Onchocerca volvulus (accession no. HG810405) from the United Kingdom [51]. In terms of Wolbachia and nematode symbiosis, it was revealed that Wolbachia are obligate mutualists for filariae worms and are necessary for the development, fertility, and vitality of adult filariae [52]. Wolbachia are found in all developmental stages of the filarial nematode but rapidly increase in number as the nematode transitions from its insect vectors to mammalian hosts. The Wolbachia titres further increase during the development of the larvae into the adult stages. The high titre of Wolbachia is also found within oocytes and infected embryos [53,54]. This study highlights PCR as the diagnostic tool for filariasis and the presence of Wolbachia in dogs from the Bangkok Metropolitan Region, Thailand. Regarding the limitations of the study, the examination of additional dog blood samples from geographical regions of Thailand is required for a more complete and robust understanding of the phylogeography of Wolbachia and filariasis in Thailand. The use of bacteria as a new control trend simultaneously targeting the vector and filarial parasites should also be explored in the future.

\section{Materials and Methods}

\subsection{Ethics Statement}

The study was approved by the animal research ethics committee of Chulalongkorn University Animal Care and Use Protocol (CU-ACUP), Faculty of Medicine, Chulalongkorn University, Bangkok, Thailand (COA No. 022/2561).

\subsection{Sample Collection}

A total of 57 blood samples were collected from domestic dogs in the Bangkok Metropolitan Region, Thailand by collaborating with veterinarians at the Small Animal Teaching Hospital, Chulalongkorn University and Faculty of Veterinary Science, Mahidol University Salaya Campus. The specimens were stored at $4{ }^{\circ} \mathrm{C}$ and transferred to the laboratory of the Vector Biology and Vector Borne Disease Research Unit, Department of Parasitology, Faculty of Medicine, Chulalongkorn University for filarial worm detection (Table S1). These samples were differentiated for morphological identification and molecular detection.

\subsection{Microscopic Examination}

Drops of blood were smeared on glass slides. Thick smears were fixed with absolute methanol (Sigma-Aldrich, St. Louis, MO, USA) for one minute, stained with Giemsa (Sigma-Aldrich, St. Louis, $\mathrm{MO}, \mathrm{USA}$ ) in a phosphate buffer (with a $\mathrm{pH}$ of 7.2), examined under a light microscope (CH20, 
Olympus, Tokyo, Japan), and morphologically identified for microfilariae with or without sheath species using the taxonomic key [55-57].

\subsection{DNA Extraction}

DNA was extracted from the dog blood samples by using a blood DNA extraction kit (Invisorb Spin Blood Mini Kit, STRATEC Molecular, Berlin, Germany) according to the manufacturer's instructions. Extracted DNA was eluted in $50 \mu \mathrm{L}$ of elution buffer. Extracted DNA samples were kept at $-80^{\circ} \mathrm{C}$ for long-term storage.

\subsection{Molecular Identification of Canine Filarial}

The microfilariae DNA samples were amplified for partial COI genes and ITS1 regions. For the COI gene, two oligonucleotide primers from previous studies [58], COI-int-F (5'-TGATTGGTGGTTT TGGTAA- $3^{\prime}$ ) and COI-int-R (5'-ATAAGTACGAGTATCAATATC- $3^{\prime}$ ), were used for PCR amplification. The PCR reactions were performed in a total volume of $20 \mu \mathrm{L}$ containing 50 ng of DNA template, $10 \times$ buffer (Thermo Fisher Scientific, Waltham, MA, USA), $25 \mathrm{mM}$ of $\mathrm{MgCl}_{2}$ (Thermo Fisher Scientific, Waltham, MA, USA), $2 \mathrm{mM}$ of dNTPs (GeneAll, Korea), $10 \mu \mathrm{M}$ each of forward and reverse primers, and five units of Taq DNA polymerase (Thermo Fisher Scientific, Waltham, MA, USA). The thermal profile was as follows: $94{ }^{\circ} \mathrm{C}$ for $3 \mathrm{~min} ; 40$ cycles of $94{ }^{\circ} \mathrm{C}$ for $45 \mathrm{~s}$, $52{ }^{\circ} \mathrm{C}$ for $45 \mathrm{~s}$, and $72{ }^{\circ} \mathrm{C}$ for $90 \mathrm{~s}$; and $72{ }^{\circ} \mathrm{C}$ for $7 \mathrm{~min}$. The ITS1 region was PCR-amplified using forward primer ITS1-F (5'-GGTGAACCTGCGGAAGGATC- $\left.3^{\prime}\right)$ and reverse primer ITS1-R (5'-CTCAATGCGTCTGCAATTCGC -3'), and this was performed in $25 \mu \mathrm{L}$ of reaction mixture containing $50 \mathrm{ng}$ of DNA template, 10× buffer (Thermo Fisher Scientific, Waltham, MA, USA), $25 \mathrm{mM}$ of $\mathrm{MgCl}_{2}$ (Thermo Fisher Scientific, MA, USA), $2 \mathrm{mM}$ of dNTPs (GeneAll, Korea), $10 \mu \mathrm{M}$ of each primer, and five units of Taq DNA polymerase (Thermo Fisher Scientific, Waltham, MA, USA). The PCR conditions were $94{ }^{\circ} \mathrm{C}$ for $5 \mathrm{~min} ; 35$ cycles of $94{ }^{\circ} \mathrm{C}$ for $30 \mathrm{~s}, 58{ }^{\circ} \mathrm{C}$ for $30 \mathrm{~s}$, and $72{ }^{\circ} \mathrm{C}$ for $45 \mathrm{~s}$; and $72{ }^{\circ} \mathrm{C}$ for $10 \mathrm{~min}$ [31]. The positive controls used in this study consisted of D. immitis, B. pahangi, and B. malayi DNA, whereas double-distilled water was used as a negative control. The PCR products were analyzed via $1.5 \%$ agarose gel electrophoresis, stained with ethidium bromide, and visualized with Quantity One Quantification Analysis Software Version 4.5.2 (Gel DocEQ System; Bio-Rad, Hercules, CA, USA).

\subsection{Wolbachia Bacterial Detection}

The nested PCR screening for Wolbachia bacteria was amplified by using two pairs of primer. Two primers, including Wol1-fwd (5'-CCTGTACTATATCCAAGAATTACTG-3') and Wol1-R (5'-AC TATCCTTTATATGTTCCATAATTTC-3), were used for the first round. For the second round of amplification, two primers, Wol7-fwd (5'-GGTGGAAATGCTGTGAATAAC-3') and Wol7-R (5'-AGC ACCGAGCCCTTTAG-3'), that were previously designed on the FtsZ region were used [59]. The PCR mixtures were $25 \mu \mathrm{L}$ in total, consisting of $50 \mathrm{ng}$ of DNA template, $10 \times$ buffer (Thermo Fisher Scientific, Waltham, MA, USA), $25 \mathrm{mM}$ of $\mathrm{MgCl}_{2}$ (Thermo Fisher Scientific, Waltham, MA, USA), $2 \mathrm{mM}$ of dNTPs (GeneAll, Korea), $300 \mathrm{nM}$ of each primer, and five units of Taq DNA Polymerase (Thermo Fisher Scientific, Waltham, MA, USA). The thermal profiles used were as described previously. The positive control for Wolbachia PCR used was the DNA of Wolbachia supergroup B in Aedes albopictus mosquitoes, while double-distilled water was used as a negative control. The PCR products were examined using $1.5 \%$ agarose gel electrophoresis, stained with ethidium bromide, and visualized under ultraviolet transilluminator Quantity One Quantification Analysis Software Version 4.5.2 (Gel DocEQ System; Bio-Rad, Hercules, CA, USA).

\subsection{Cloning and Sequencing}

All positive PCR amplicons were ligated into pGEM-T Easy Vector (Promega, Madison, WI, USA), and the recombinant plasmids were used to transform a competent Escherichia coli DH5 $\alpha$ strain. 
Transformed cells were cultured and recombinant plasmids were then extracted using Invisorb ${ }^{\circledR}$ Spin Plasmid Mini kit (STRATEC Molecular, Berlin, Germany) following the manufacturer's instructions. Plasmids were sequenced by a commercial service at AITBIOTECH, Singapore and MACROGEN, Korea.

\subsection{Sequence Analysis and Phylogenetic Tree Construction}

The sequences were aligned using BioEdit Sequence Alignment Editor Version 7.2.5 [60]. The phylogenetic trees were constructed using the maximum-likelihood method with IQ-TREE on the IQ-TREE web server (http://iqtree.cibiv.univie.ac.at/) with 1000 ultrafast bootstrap replicates. The best-fit model of substitution was found using the auto function on the IQ-TREE web server [61]. The phylogenetic tree was finally viewed and edited with FigTree v1.4.4 software (http://tree.bio.ed.ac.uk/software/figtree/).

\section{Conclusions}

Between the aforementioned tested PCR methods, the COI-based method was more suitable for diagnosing canine filaria than the ITS1-based method. This COI-PCR method could differentiate D. immitis, B. malayi, and B. pahangi by their amplicon sizes in a single-tube PCR. This method could be useful for the epidemiological survey of filarial infection in humans, mosquitoes, and other reservoirs in Thailand. In addition, the detection of Wolbachia within filaria-infected dogs in our locality shows the potential of using the bacteria as a new control trend to be done simultaneously with targeting the vector and filarial parasites.

Supplementary Materials: The following are available online at http://www.mdpi.com/2076-0817/8/3/114/s1, Figure S1: PCR amplification of the partial COI gene for filarial nematode, Figure S2: PCR amplification of the ITS1 region for the filarial nematode, Figure S3: Sequence alignment of the filaria nematode based on the partial COI gene, Figure S4: Sequence alignment of the filaria nematode based on the ITS1 region, Figure S5: PCR amplicons of the FtsZ specific to Wolbachia, Figure S6: Sequence alignment of Wolbachia bacteria based on the FtsZ gene, Table S1: Raw data of samples.

Author Contributions: Conceptualization, K.P. and P.S.; methodology, H.S.; software, H.S. and A.P.; validation, K.P., P.S., and H.S.; formal analysis, K.P.; investigation, A.P.; resources, S.T. and S.S.; data curation, P.S. and K.P.; writing — original draft preparation, H.S. and A.P.; writing-review and editing, K.P., P.S., A.P., and N.B.; visualization, P.S.; supervision, K.P.; project administration, H.S.; funding acquisition, K.P. and P.S.

Funding: This study was supported by the Ratchadapiseksompotch Fund (grant no. RA.62/021), the Faculty of Medicine, Chulalongkorn University, National Research Council of Thailand and Chulalongkorn University (grant no. GRB_APS11593010), and the Research Assistant Scholarship, Chulalongkorn University.

Acknowledgments: We would like to thank the staff of Vector Biology and Vector Borne Disease Research Unit, Department of Parasitology, Faculty of Medicine, Chulalongkorn University, Small Animal Teaching Hospital, Chulalongkorn University, and Faculty of Veterinary Science, Mahidol University Salaya Campus, Thailand.

Conflicts of Interest: The authors declare no conflict of interest.

\section{References}

1. World Health Organization. Lymphatic Filariasis. Available online: https://www.who.int/news-room/factsheets/detail/lymphatic-filariasis (accessed on 28 May 2019).

2. Kaikuntod, M.; Thongkorn, K.; Tiwananthagorn, S.; Boonyapakorn, C. Filarial worms in dogs in Southeast Asia. Vet. Integr. Sci. 2018, 16, 1-17.

3. Mak, J.W.; Yen, P.K.; Lim, K.C.; Ramiah, N. Zoonotic implications of cats and dogs in filarial transmission in Peninsular Malaysia. Trop. Geogr. Med. 1980, 32, 259-264.

4. Irwin, P.J. Companion animal parasitology: A clinical perspective. Int. J. Parasitol. Res. 2002, 32, 581-593. [CrossRef]

5. Kelly, J.D. Canine heart worm disease. In Current Veterinary Therapy VII; W. B. Saunders: Philadelphia, PA, USA, 1979; pp. 326-335.

6. Rishniw, M.; Barr, S.C.; Simpson, K.W.; Frongillo, M.F.; Franz, M.; Dominguez Alpizar, J.L. Discrimination between six species of canine microfilariae by a single polymerase chain reaction. Vet. Parasitol. 2006, 135, 303-314. [CrossRef] 
7. Kanjanopas, K.; Choochote, W.; Jitpakdi, A.; Suvannadabba, S.; Loymak, S.; Chungpivat, S.; Nithiuthai, S. Brugia malayi in a naturally infected cat from Narathiwat province, southern Thailand. Southeast. Asian J. Trop. Med. Public Health 2001, 32, 585-587.

8. Kamyingkird, K.; Junsiri, W.; Chimnoi, W.; Kengradomkij, C.; Saengow, S.; Sangchuto, K.; Ka jeerum, W.; Pangjai, D.; Nimsuphan, B.; Inpankeaw, T.; et al. Prevalence and risk factors associated with Dirofilaria immitis infection in dogs and cats in Songkhla and Satun provinces, Thailand. Agric. Nat. Resour. 2017, 51, 299-302. [CrossRef]

9. Wongkamchai, S.; Nochotea, H.; Foongladda, S.; Dekumyoy, P.; Thammapalo, S.; Boitanoa, J.J.; Choochotee, W. A high resolution melting real time PCR for mapping of filaria infection in domestic cats living in brugian filariosis-endemic areas. Vet. Parasitol. 2014, 201, 120-127. [CrossRef]

10. Simón, F.; Morchón, R.; González-Miguel, J.; Marcos-Atxutegi, C.; Siles-Lucas, M. What is new about animal and human dirofilariosis? Trends. Parasitol. 2009, 25, 404-409. [CrossRef]

11. Ichimori, K.; King, J.D.; Engels, D.; Yajima, A.; Mikhailov, A.; Lammie, P.; Ottesen, E.A. Global programme to eliminate lymphatic filariasis: The processes underlying programme success. PLoS. Negl. Trop. Dis. 2014, 8, e3328. [CrossRef]

12. World Health Organization. Essential Medicines Donated to Control, Eliminate and Eradicate Neglected Tropical Diseases; World Health Organization: Geneva, Switzerland, 2017.

13. World Health Organization. Lymphatic Filariasis: A Handbook of Practical Entomology for National Lymphatic Filariasis Elimination Programmes; World Health Organization: Geneva, Switzerland, 2013.

14. Famakinde, D.O. Mosquitoes and the Lymphatic Filarial Parasites: Research Trends and Budding Roadmaps to Future Disease Eradication. Trop. Med. Infect. Dis. 2018, 43, 4. [CrossRef]

15. Pradatsundarasar, A. Dirofilaria infection in man: Report of a case. J. Med. Assoc. Thailand 1955, 38, 378-379.

16. Jariya, P.; Sucharit, S. Dirofilaria repens from the eyelid of a woman in Thailand. Am. J. Trop. Med. Hyg. 1983, 32, 1456-1457. [CrossRef]

17. Sukudom, P.; Phumee, A.; Siriyasatien, P. First report on subconjunctival dirofilariasis in Thailand caused by a Dirofilaria sp. closely related to D. hongkongensis. Acad. J. Sci. Res. 2018, 6, 114-116. [CrossRef]

18. Taylor, M.J.; Bandi, C.; Hoerauf, A. Wolbachia bacterial endosymbionts of filarial nematodes. Adv. Parasitol. 2005, 60, 245-284. [CrossRef]

19. Taylor, M.J.; Voronin, D.; Johnston, K.L.; Ford, L. Wolbachia filarial interactions. Cell. Microbiol. 2013, 15, 520-526. [CrossRef]

20. Bandi, C.; Trees, A.J.; Brattig, N.W. Wolbachia in filarial nematodes: Evolutionary aspects and implications for the pathogenesis and treatment of filarial diseases. Vet. Parasitol. 2001, 98, 215-238. [CrossRef]

21. Werren, J.H.; Baldo, L.; Clark, M.E. Wolbachia: Master manipulators of invertebrate biology. Nat. Rev. Microbiol. 2008, 6, 741-751. [CrossRef]

22. Clark, E.L.; Karley, A.J.; Hubbard, S.F. Insect endosymbionts: Manipulators of insect herbivore trophic interactions? Protoplasma 2010, 244, 25-51. [CrossRef]

23. Slatko, B.E.; Taylor, M.J.; Foster, J.M. The Wolbachia endosymbiont as an anti-filarial nematode target. Symbiosis 2010, 51, 55-65. [CrossRef]

24. Pfarr, K.M.; Hoerauf, A.M. Antibiotics which target the Wolbachia endosymbionts of filarial parasites: A new strategy for control of filariasis and amelioration of pathology. Mini. Rev. Med. Chem. 2006, 6, 203-210. [CrossRef]

25. Ravindran, R.; Varghese, S.; Nair, S.N.; Balan, V.M.; Lakshmanan, B.; Ashruf, R.M.; Kumar, S.S.; Gopalan, A.K.; Nair, A.S.; Malayil, A.; et al. Canine filarial infections in a human Brugia malayi endemic area of India. Biomed. Res. Int. 2014, 2014, 630160. [CrossRef]

26. Megat Abd Rani, P.A.; Irwin, P.J.; Gatne, M.; Coleman, G.T.; McInnes, L.M.; Traub, R.J. A survey of canine filarial diseases of veterinary and public health significance in India. Parasit. Vectors 2010, 3, 30. [CrossRef]

27. Saseendranath, M.R.; Varghese, C.G.; Jayakumar, K.M. Incidence of canine dirofilariosis in Trichur, Kerala. Indian J. Vet. Med. 1986, 6, 139.

28. Otranto, D.; Dantas-Torres, F.; Breitschwerdt, E.B. Managing canine vector-borne diseases of zoonotic concern: Part one. Trends Parasitol. 2009, 25, 157-163. [CrossRef]

29. Rosenblatt, J.E. Laboratory diagnosis of infections due to blood and tissue parasites. Clin. Infect. Dis. 2009, 49, 1103-1108. [CrossRef] 
30. Ricciardi, A.; Ndao, M. Diagnosis of Parasitic Infections: What's Going On? J. Biomol. Screen. 2015, 20,6 -21. [CrossRef]

31. Nuchprayoon, S.; Junpee, A.; Poovorawan, Y.; Scott, A.L. Detection and differentiation of filarial parasites by universal primers and polymerase chain reaction-restriction fragment length polymorphism analysis. Am. J. Trop. Med. Hyg. 2005, 73, 895-900. [CrossRef]

32. Magnis, J.; Lorentz, S.; Guardone, L.; Grimm, F.; Magi, M.; Naucke, T.J.; Deplazes, P. Morphometric analyses of canine blood microfilariae isolated by the Knott's test enables Dirofilaria immitis and D. repens species-specific and Acanthocheilonema (syn. Dipetalonema) genus-specific diagnosis. Parasit. Vectors 2013, 6, 48. [CrossRef]

33. Hoch, H.; Strickland, K. Canine and feline dirofilariasis: Life cycle, pathophysiology, and diagnosis. Compend. Contin. Educ. Vet. 2008, 30, 133-140.

34. Casiraghi, M.; Bazzocchi, C.; Mortarino, M.; Ottina, E.; Genchi, C. A simple molecular method for discriminating common filarial nematodes of dogs (Canis familiaris). Vet. Parasitol. 2006, 141, 368-372. [CrossRef]

35. Wang, S.; Zhang, N.; Zhang, Z.; Wang, D.; Yao, Z.; Zhang, H.; Ma, J.; Zheng, B.; Ren, H.; Liu, S. Prevalence of Dirofilaria immitis infection in dogs in Henan province, central China. Parasite 2016, 23, 43. [CrossRef]

36. Byeon, K.H.; Kim, B.J.; Kim, S.M.; Yu, H.S.; Jeong, H.J.; Ock, M.S. A serological survey of Dirofilaria immitis infection in pet dogs of Busan, Korea, and effects of chemoprophylaxis. Korean J. Parasitol. 2007, 45, 27-32. [CrossRef]

37. Khedri, J.; Radfar, M.H.; Borji, H.; Azizzadeh, M.; Akhtardanesh, B. Canine Heartworm in Southeastern of Iran with Review of disease distribution. Iran J. Parasitol. 2014, 9, 560-567.

38. Alho, A.M.; Landum, M.; Ferreira, C.; Meireles, J.; Goncalves, L.; de Carvalho, L.M.; Belo, S. Prevalence and seasonal variations of canine dirofilariosis in Portugal. Vet. Parasitol. 2014, 206, 99-105. [CrossRef]

39. Bacsadi, A.; Papp, A.; Szeredi, L.; Toth, G.; Nemes, C.; Imre, V.; Tolnai, Z.; Szell, Z.; Sreter, T. Retrospective study on the distribution of Dirofilaria immitis in dogs in Hungary. Vet. Parasitol. 2016, 220, 83-86. [CrossRef]

40. Tiawsirisup, S.; Thanapaisarnkit, T.; Varatorn, E.; Apichonpongsa, T.; Bumpenkiattikun, N.; Rattanapuchpong, S.; Chungpiwat, S.; Sanprasert, V.; Nuchprayoon, S. Canine Heartworm (Dirofilaria immitis) Infection and Immunoglobulin G Antibodies Against Wolbachia (Rickettsiales: Rickettsiaceae) in Stray Dogs in Bangkok, Thailand. Thai. J. Vet. Med. 2015, 40, 165-170.

41. Ambily, V.R.; Pillai, U.N.; Arun, R.; Pramod, S.; Jayakumar, K.M. Detection of human filarial parasite Brugia malayi in dogs by histochemical staining and molecular techniques. Vet. Parasitol. 2011, 181, 210-214. [CrossRef]

42. Chungpivat, S.; Taweethavonsawat, P. The differentiation of microfilariae in dogs and cats using Giemsa's staining and the detection of acid phosphatase activity. J. Thai Vet. Pract. 2008, 20, 47-55.

43. Thanchomnang, T.; Intapan, P.M.; Chungpivat, S.; Lulitanond, V.; Maleewong, W. Differential detection of Brugia malayi and Brugia pahangi by real-time fluorescence resonance energy transfer PCR and its evaluation for diagnosis of B. pahangi-infected dogs. Parasitol. Res. 2010, 106, 621-625. [CrossRef]

44. Chansiri, K.; Tejangkura, T.; Kwaosak, P.; Sarataphan, N.; Phantana, S.; Sukhumsirichart, W. PCR based method for identification of zoonostic Brugia malayi microfilariae in domestic cats. Mol. Cell. Probes 2002, 16, 129-135. [CrossRef]

45. Oh, I.Y.; Kim, K.T.; Sung, H.J. Molecular Detection of Dirofilaria immitis Specific Gene from Infected Dog Blood Sample Using Polymerase Chain Reaction. Iran. J. Parasitol. 2017, 12, 433-440.

46. Lo, N.; Casiraghi, M.; Salati, E.; Bazzocchi, C.; Bandi, C. How many Wolbachia supergroups exist? Mol. Biol Evol. 2002, 19, 341-346. [CrossRef]

47. Casiraghi, M.; Bordenstein, S.R.; Baldo, L.; Lo, N.; Beninati, T.; Wernegreen, J.J.; Werren, J.H.; Bandi, C. Phylogeny of Wolbachia pipientis based on gltA, groEL and ftsZ gene sequences: Clustering of arthropod and nematode symbionts in the F supergroup, and evidence for further diversity in the Wolbachia tree. Microbiology 2005, 151, 4015-4022. [CrossRef]

48. Bordenstein, S.R.; Paraskevopoulos, C.; Hotopp, J.C.; Sapountzis, P.; Lo, N.; Bandi, C.; Tettelin, H.; Werren, J.H.; Bourtzis, K. Parasitism and mutualism in Wolbachia: What the phylogenomic trees can and cannot say. Mol. Biol. Evol. 2009, 26, 231-241. [CrossRef]

49. Baldo, L.; Werren, J.H. Revisiting Wolbachia supergroup typing based on WSP: Spurious lineages and discordance with MLST. Curr. Microbiol. 2007, 55, 81-87. [CrossRef] 
50. Bandi, C.; Anderson, T.J.; Genchi, C.; Blaxter, M.L. Phylogeny of Wolbachia in filarial nematodes. Proc. Biol. Sci. 1998, 265, 2407-2413. [CrossRef]

51. Darby, A.C.; Armstrong, S.D.; Bah, G.S.; Kaur, G.; Hughes, M.A.; Kay, S.M.; Koldkjær, P.; Rainbow, L.; Radford, A.D.; Blaxter, M.L.; et al. Analysis of gene expression from the Wolbachia genome of a filarial nematode supports both metabolic and defensive roles within the symbiosis. Genome Res. 2012, 22, 2467-2477. [CrossRef]

52. Lefoulon, E.; Bain, O.; Makepeace, B.L.; d'Haese, C.; Uni, S.; Martin, C.; Gavotte, L. Breakdown of coevolution between symbiotic bacteria Wolbachia and their filarial hosts. PeerJ 2016, 4, e1840. [CrossRef]

53. Fenn, K.; Blaxter, M. Quantification of Wolbachia bacteria in Brugia malayi through the nematode lifecycle. Mol. Biochem. Parasitol. 2004, 137, 361-364. [CrossRef]

54. McGarry, H.F.; Egerton, G.L.; Taylor, M.J. Population dynamics of Wolbachia bacterial endosymbionts in Brugia malayi. Mol. Biochem. Parasitol. 2004, 135, 57-67. [CrossRef]

55. Nelson, G.S. The identification of infective filarial larvae in mosquitoes: With a note on the species found in "wild" mosquitoes on the Kenya coast. J. Helmintho. 1959, 33, 233-256. [CrossRef]

56. Yen, P.K.F.; Zaman, V.; Mak, J.W. Identification of some common infective filarial larvae in Malaysia. J. Helminthol. 1982, 56, 69-80. [CrossRef]

57. Orihel, T.C.; Ash, L.R.; Ramachandran, C.P.; Ottesen, E.A. Bench Aids for the Diagnosis of Filarial Infections; World Health Organization: Geneva, Switzerland, 1997.

58. Casiraghi, M.; Anderson, T.J.; Bandi, C.; Bazzocchi, C.; Genchi, C. A phylogenetic analysis of filarial nematodes: Comparison with the phylogeny of Wolbachia endosymbionts. Parasitology 2001, 122, 93-103. [CrossRef]

59. Turba, M.E.; Zambon, E.; Zannoni, A.; Russo, S.; Gentilini, F. Detection of Wolbachia DNA in blood for diagnosing filaria-associated syndromes in cats. J. Clin. Microbiol. 2012, 50, 2624-2630. [CrossRef]

60. Hall, T.A. BioEdit: A user-friendly biological sequence alignment editor and analysis program for Windows 95/98/NT. Nucleic. Acids. Symp. Ser. 1999, 41, 95-98.

61. Trifinopoulos, J.; Nguyen, L.T.; von Haeseler, A.; Minh, B.Q. W-IQ-TREE: A fast online phylogenetic tool for maximum likelihood analysis. Nucleic. Acids. Res. 2016, 44, W232-W235. [CrossRef]

(C) 2019 by the authors. Licensee MDPI, Basel, Switzerland. This article is an open access article distributed under the terms and conditions of the Creative Commons Attribution (CC BY) license (http://creativecommons.org/licenses/by/4.0/). 